\title{
RESPUESTAS DOCENTES SOBRE EL CINE COMO PROPUESTA PEDAGÓGICA. ANÁLISIS DE LA SITUACIÓN EN EDUCACIÓN SECUNDARIA
}

\author{
Educational answers on the cinema like pedagogic \\ proposals. Descriptive analysis of the situation in the \\ Secundary Education
}

\section{M. ${ }^{a}$ Carmen PEREIRA DOMÍNGUEZ y M. ${ }^{a}$ Victoria MARÍN VALLE \\ Universidad de Vigo. Facultad de Ciencias de la Educación. Departamento de. Análisis e Intervención Psicosocioeducativa. Campus de Ourense. Avenida de Castelao,s/n.32004 Ourense.modguez@uvigo.es}

Fecha de aceptación definitiva: marzo de 2002

BIBLID [(1130-3743) 13, 2001, 233-255]

\section{RESUMEN}

Esta investigación analiza los recursos audiovisuales y el uso que hace el profesorado del primer ciclo de Enseñanza Secundaria Obligatoria de los medios audiovisuales, en especial del cine, dentro de sus tareas educativas.

En este sentido, se estudian las razones por las cuales se utiliza o no el cine en las aulas, así como la percepción de los docentes con respecto al tema y el uso, el dominio y la disposición ante el cine forum, como estrategia de intervención pedagógica.

De las conclusiones principales extraídas del estudio, resaltamos la positiva predisposición del profesorado ante este tipo de medios, a pesar de las dificultades detectadas para su uso, así como la necesidad de establecer vías de formación permanente y de elaborar materiales adecuados que faciliten la inclusión de estos medios en los procesos de aprendizaje.

Palabras clave: actitudes, cine, educación, educación audiovisual, intervención pedagógica, profesorado Secundaria. 
This investigation analyses the audiovisual resources and the use that he/she makes the faculty of the first cycle of Obligatory Secondary Education of the audiovisual means, especially of the cinema, inside its educational tasks.

In this way, the reasons are studied by which it is used or not the cinema in the classrooms, as well as the perception of the educational ones with regard to the topic and the use, the domain and the disposition before the cinema forum, as strategy of pedagogic intervention.

Of the extracted main conclusions of the study, we stand out the faculty's positive bias before this type of means, in spite of the difficulties detected for their use, as well as the necessity to establish roads of permanent formation and of elaborating appropriate materials that facilitate the inclusion of these means in the learning processes.

Key words: attitude, audiovisual education, cinema, education, pedagogic intervention, secundary teaching.

El artículo informa de una investigación sobre la utilización del cine como medio recurso de enseñanza-aprendizaje en la Enseñanza Secundaria Obligatoria. En ella se perseguirá un doble objetivo:

1. Conocer y diagnosticar la situación actual en lo que respecta a la utilización de estas herramientas pedagógicas.

2. A partir de los resultados hacer propuestas encaminadas a potenciar y mejorar su utilización desde una concepción integradora del proceso educativo.

Para ello, se construyó en primer lugar un marco conceptual que, como tal, sirva de fundamento para el diseño de la investigación y la elaboración del instrumento de recogida de datos (cuestionario). Una vez analizados éstos y extraídas las conclusiones, proponemos alternativas de mejora.

\section{MARCO TEÓRICO: EL CiNE Y LA EDUCACIÓN}

Al gran número de medios de información y comunicación de carácter social que hoy en día proliferan los hemos denominado en su conjunto mass-media. No cabe duda de que la tendencia a considerar estos medios es cada vez mayor, más aún si tenemos en cuenta la cantidad de información que nos proporcionan dentro de las más variadas tendencias ideológicas. Desde este punto de vista nos encontramos ante el reto, desde el mundo educativo, de saber, con espíritu crítico, analizar tanto el "qué se dice" como el "cómo se dice". Así entendido, no cabe duda que hoy en día la importancia de la imagen en el mundo adolescente es algo que a nadie se le escapa. 
M. a CARMEN PEREIRA DOMÍNGUEZ Y M. VICTORIA MARÍN VALLE

Así el cine como instrumento de comunicación se nos presenta en el ámbito educativo como una herramienta fascinante de trabajo pedagógico con los alumnos. Un medio audiovisual como puede ser el cine, entendido éste como aquella forma de instrucción en la que se utilizan los oídos, los ojos, o ambos sentidos al mismo tiempo, proporciona a la educación el método intuitivo adecuado para afianzar contenidos nuevos a la vez que se desarrollan capacidades y habilidades importantes para el individuo.

La confianza que han depositado las actuales tendencias de renovación pedagógica en el poder formativo del cine hace que stu presencia en la escuela vaya adquiriendo paulatinamente nuevas dimensiones. Si retrocedemos en el tiempo, comprobamos que el cine es el único arte que han visto nacer las generaciones presentes; surgió entre el interés de unos pocos y la indiferencia o desprecio de la mayoría. Primero, se constituyó como una curiosidad de la física recreativa y posteriormente pasó a espectáculo de barraca de feria (Gubern, 1995).

Durante muchos años el cine buscó solución a sus problemas de estética y narrativa, intentando crear un lenguaje propio, así como a través de la perfección técnica. Su rápida evolución permitió juzgarle y valorarle con cierta moderación, hasta que en el año 1911 Ricciotto Canudo lo catalogó como el séptimo arte situándolo junto a la literatura, pintura, música, danza, escultura y arquitectura (Jeanne y Ford, 1988; De la Torre, 1996; Espelt, 2001). Como consecuencia, el cine deja de considerarse la vía de entretenimiento típico de fiesta, como antaño, para constituirse en lenguaje universal. Como tal, el sistema educativo aprovecha las propiedades del cine formativo para impregnar las áreas del currículum de educación primaria y secundaria ${ }^{1}$. Por todo ello, el actual alumnado necesita convertirse en "... alguien capaz de afrontar, desde el conocimiento y la experiencia, el tremendo aluvión audiovisual que cada vez de forma más agresiva lo ataca más insistentemente desde todas las esquinas que deberá sortear en su trayecto..." (González Martel, 1996, 23).

Si uno de los principios básicos con más fuerza en la actualidad es la vinculación entre comunidad educativa y sociedad, la escuela no puede mantenerse ajena a esta realidad, es más, debe acortar distancias entre la vida de los escolares y el sistema educativo. Para eso, el cine no sólo ha de estar presente en la escuela, sino que además demanda conocimientos en continua actualización para su adecuada comprensión y utilización. Esto significa no considerarlo como un parche alternativo dentro del proceso de enseñanza-aprendizaje del alumnado, sino como una porción integrante de todo el currículo (Coll, Selva y Solá, 1995). Es más,

1. La presencia del cine en los actuales programas de enseñanza se encuentra, por una parte, en las áreas del currículum de Educación Primaria y Secundaria, concretamente, en las correspondientes a lengua castellana y literatura, conocimiento del medio, ciencias sociales y educación artística, y por otra parte, como componente de dos materias optativas: "Imagen y expresión" y "Los procesos de comunicación". 
Si apostamos por un currículum donde el cine forma parte de un sistema coherente multidisciplinar, transversalizado e integrado en el que se articulan: objetivos, procesos de aprendizaje, contenidos explícitos y latentes, desarrollo de evaluación, así como su organización, secuencialización y temporalización, es porque creemos que aprender con, por y desde el cine implica introducir contenidos y estrategias de aprendizaje que se deben ir desarrollando, según los sucesivos niveles de profundización, no sólo hasta el final de la Enseñanza Secundaria, sino a lo largo de toda la vida (Alonso y Pereira, 2000: 132).

Ante lo expuesto, abogamos por la idea de concebir el cine no sólo como un medio de comunicación sin más, sino como una herramienta pedagógica permanente en los procesos de aprendizaje del alumnado que promueve el desarrollo de habilidades sociales, además de predisponer a la reflexión, al análisis y al juicio crítico, así como también a crear y a transmitir actitudes y valores sociales y culturales (Vázquez Gómez, 1994; Platas, 1994; Dios, 2001). Su potencialidad como elemento transmisor de conceptos, valores y pautas de conducta se ha hecho patente desde sus orígenes (Coppen, 1991; Lumet, 1999). El cine posee una innegable influencia en los valores de la sociedad. Se convierte en cierto modo en altavoz de valores, de creencias y comportamientos de masas que se encuentran poco habituadas a recrear el pensamiento crítico. De ahí que nuestra función como educadores consista en marcar caminos idóneos para que cultura y sujeto caminen juntos para establecer formas de conocer y enfocar la realidad hacia la línea epistemológica organizada que no sólo permita la reproducción del orden cultural sino también la reconstrucción reflexiva y crítica.

Merece destacarse la tesis de Ying cuando confirmaba que "el cine es un reflejo de la realidad social en la que vivimos" $(1987,13)$. En esta misma línea, también Bodgan y Biklen subrayan que "desde una perspectiva psicosocial y antropológica, puede afirmarse que los estereotipos llevan, muy frecuentemente, incluidos valores y conceptos ideales anclados en lo que podría llamarse la mente colectiva" $(1982,5)$. Por ello, se considera que los valores latentes en la realidad actual se reflejan en los filmes y se pueden leer a través de los diálogos y de las imágenes. Desde el lenguaje cinematográfico, el director puede expresar una manifestación sobre qué son los planteamientos éticos y las concepciones ideológicas que se encuentran arraigadas dentro de lo que constituye la conciencia social.

El cine merece ser un espacio formativo, conviene pues aprovechar su potencialidad formativa y adoptar una actitud positiva frente a la idea mayoritariamente entendida hoy día sobre él, es decir, como inductor de acción, violencia y sexo (Rodríguez Neira, 1999; Loscertales y Núñez, 2001). Por la frescura que aún conserva, nos parece notorio citar en este espacio algunas ideas de las conclusiones extraídas en el Primer Congreso Democrático del Cine Español, celebrado en 1979, donde se resaltaba que "El cine debe ocupar en los centros docentes el lugar que le corresponde como hecho cultural de primera magnitud, tratando de hacer que desaparezca el carácter que se le ha dado de mero entretenimiento y resaltando sus valores educativos y culturales» (Fernández Sebastián, 1989, 5). Años más tarde, es 
M. CARMEN PEREIRA DOMINGUEZ Y M.` VICTORIA MARÍN VALLE

frecuente descubrir el cine además de elemento de aprendizaje, como una alternativa de ocio y recreación personal (Lamet, 1991).

Creemos que el concepto cine formativo es más que una expresión de moda. Refleja una nueva forma de percibirlo desde una vertiente educativa. Consiste en la emisión y recepción intencional de películas con cierto reconocimiento cinematográfico y que pueden ser analizadas desde una óptica pedagógica en orden a promover valores (Escámez y Ortega, 1989; Buxarrais, Martínez, Puig y Tri1la, 1995; Varios, 1997; Ortega, Gil y Mínguez, 1997; Vázquez Freire, 1999). Por consiguiente, consideramos de interés adoptar el enunciado que sobre este concepto defiende Saturnino de la Torre:

...cine formativo es la emisión y recepción intencional de películas portacloras de valores culturales, humanos, técnico-científicos o artísticos, con la finalidad de mejorar el conocimiento, las estrategias o las actitudes y opiniones de los espectadores. En primer lugar, ha de poseer determinados valores o méritos humanos; en segundo lugar, dichos valores han de estar codificados de forma que sean interpretables y en tercer lugar que exista una intención de utilizar dicho medio como recurso formativo, es decir, con voluntad de producir algún tipo de aprendizaje, que no tiene por qué estar vinculado necesariamente al mensaje de la película... $(1996,17)$.

En definitiva, se trata de películas que ayuden al crecimiento personal y profesional de los ciudadanos. Además, si se estima el cine como una obra artística, se piensa de modo inmediato que éste provoca motivación, incitación y sensibilización hacia los valores sociales y culturales que conlleva (Urpí, 2000). Aunque en la actualidad el cine se entremezcla con los acontecimientos de la vida cotidiana, no implica que conozcamos toda su estructura básica de funcionamiento. Además el hecho de contemplar películas no supone aprovechar todas sus posibilidades, aunque también es cierto que la comprensión del cine requiere su aproximación continua, especialmente si se intenta lograr el entendimiento del mundo cinematográfico. Dada la existencia de este desconocimiento generalizado, se precisa adiestramiento y, sobre todo, formación en la reflexión, en el conocimiento y en el juicio crítico, porque "...leer un film significa conocer lo que éste dice, o, si se prefiere, entrar en comunicación con la mentalidad del autor a través de su obra. Un film es un producto de un hombre que pretende comunicar, decir algo a los demás y descubrirlo, es un paso ineludible y previo a cualquier cuestión artística, histórica, social, cultural o política sobre el cine" (Alcover y Úrbez, 1976, 23). Y avanzando un poco más, diríamos que no sólo consiste en entender el mensaje del film, sino también criticar, reaccionar, transformar todo lo que reporta.

Por todo ello, podemos afirmar que el cine se vincula a la educación y además es formativo porque, en esencia, es un lenguaje icónico que a lo largo de toda la humanidad ha conseguido una trascendencia capital. De ahí que, hoy en día, este medio alcance gran repercusión científica y social. En este sentido, necesita de la 
M. ․ CARMEN PEREIRA DOMÍNGUEZ Y M. ․ VICTORIA MARÍN VALLE RESPUESTAS DOCENTES SOBRE EL CINE COMO PROPUESTA PEDAGÓGICA. ANÁLISIS DE LA SITUACIÓN EN EDUCACIÓN SECUNDARIA

educación ya que es en parte lenguaje y en parte comunicación y, desde luego, capacitación para la comprensión de toda la riqueza comunicativa que tiene el cine.

Motivados por la cuestión concreta de este medio, nos pareció oportuno iniciar una investigación ${ }^{2}$, cuya temática se centró en constatar el conocimiento, tratamiento y utilización del cine por parte de los profesores que impartían docencia en el primer ciclo de la Educación Secundaria Obligatoria (ESO), en los centros escolares de la ciudad de Ourense. Precisamente, se trató de indagar, entre otros aspectos, la comprensión y el uso que el profesorado hacía de los medios audiovisuales, y en especial, del cine dentro de sus tareas educativas. Además, se pretendió detectar las razones por las cuales se utiliza o no el cine en las aulas, así como averiguar la actitud de los docentes con respecto al tema, y también comprobar el conocimiento, desarrollo, uso y predisposición ante la técnica del cine forum, como estrategia de intervención pedagógica.

\section{Diseño de la inVESTigación}

La investigación fue realizada con el fin de conocer la intervención educativa de los medios audiovisuales, en especial, del cine en la docencia del primer ciclo de la ESO, con la intención de optimizarla.

Para la obtención de los datos se aplicó un cuestionario, elaborado expresamente. Éste recogía información de las distintas variables que configuraban el entramado del presente trabajo. Dicho cuestionario se entregó personalmente a una muestra de 139 docentes que trabajaban en centros educativos, tanto de carácter público como privado y privado concertado, pertenecientes a la ciudad de Ourense. El número de profesores que compone la muestra representa el $60 \%$ de la población de estudio, por ello, los resultados obtenidos son considerados como significativos de la realidad donde los docentes desempeñan su profesión, es decir, en el primer ciclo de la ESO.

El proceso metodológico seguido en la investigación estableció una serie de fases donde se determinaban las funciones correspondientes a cada una de ellas (Elliot, 1990; Cohen y Manión, 1990):

1. - Fase: Preparación de la investigación. Engloba la revisión y la búsqueda bibliográfica; la acotación del problema de estudio y la definición y selección de la muestra.

2. Estudio perteneciente al Proyecto de Investigación titulado La Educación Secundaria en el ámbito gallego. La herencia del pasado y el reto del presente, concedido por la Universidad de Vigo, correspondiente al año 2000 (64102G008). Asimismo, dicho estudio se integra dentro de una línea de investigación de doctorado.

(C) Ediciones Universidad de Salamanca

Teor. educ. 13, 2001, pp. 233-255 


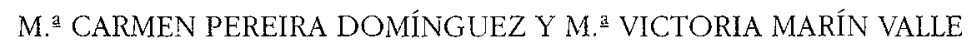

2. ${ }^{\text {a }}$ Fase: Construcción y aplicación del cuestionario. Comprende los primeros borradores del instrumento; el estudio piloto; su elaboración definitiva; la consulta a expertos y la distribución y aplicación de los cuestionarios.

3. a Fase: Recogida y análisis de la información. Incluye los 139 cuestionarios recibidos; la codificación de los datos y el análisis del contenido de las respuestas de índole abiertas y cerradas.

4. ${ }^{\text {a }}$ Fase: Preparación del informe. Contiene las conclusiones y la elaboración del informe.

Esta investigación se propuso responder a las siguientes preguntas:

- ¿Cuál es la situación sobre el uso del cine en la docencia del primer ciclo de la Educación Secundaria Obligatoria en los centros escolares de Ourense capital?

- ¿Cuál es la utilización y valoración que de él hacen los educadores que imparten docencia en este nivel?

\section{OBJETIVOS E HIPÓTESIS DE LA INVESTIGACIÓN}

Una vez planteado y ubicado el problema general, se procedió a la formulación de objetivos:

- Objetivo general:

- Constatar la situación actual del cine (conocimiento, uso y valoración) en los centros escolares de la ciudad de Ourense, mediante la apreciación que poseen los docentes en el primer ciclo de la ESO.

- Objetivos específicos:

- Conocer el grado y tipo de utilización de los medios audiovisuales que tienen los docentes del primer ciclo de la ESO.

- Averiguar las razones por las cuales se utiliza, o no, el cine en las aulas.

- Comprobar la actitud adoptada por los profesores de este nivel con respecto al uso del cine.

- Analizar si existen divergencias en la utilización del cine en comparación con otras variables correspondientes a: diversas áreas; diferentes géneros; variados tipos de centros públicos, privados y privados concertados, y distintos intervalos de edad.

- Indagar el uso, el dominio y la actitud de los profesores con relación a la técnica del cine forum.

- Enumerar las películas utilizadas y señalar otras conocidas.

De acuerdo con estos objetivos, las hipótesis del estudio quedan enunciadas de la siguiente manera. 

ANÁLISIS DE LA SITUACIÓN EN EDUCACIÓN SECUNDARIA

\section{- Hipótesis conceptual:}

La utilización de los medios audiovisuales en general, y del cine en particular, es muy escasa dentro de la práctica docente.

\section{- Hipótesis operativas:}

- No existen diferencias significativas en cuanto a la utilización del cine, en los distintos centros, ni en el género, ni en la edad.

- Se requiere una formación específica para el uso y trabajo del cine en el aula.

- Los docentes se interesan por la adecuada formación pedagógica sobre cine.

- El cine forum, como estrategia de intervención pedagógica en el aula, es poco frecuente.

- Los docentes valoran el cine como un medio que favorece la adquisición de conocimientos, de hábitos y la mejora de actitudes.

- El profesorado constata que la aplicación de la dinámica grupal del cine forum fomenta el desarrollo de las habilidades sociales.

- Las películas elegidas para la puesta en marcha de esta investigación, apenas son trabajadas de modo asiduo por los docentes del primer ciclo de la ESO.

\section{MUESTRA}

En este estudio se ha seleccionado una muestra relativa a un grupo de profesores que imparten docencia en el primer ciclo de la Educación Secundaria, en Ourense ciudad, esto es, el universo de población lo constituyen un total de 228 sujetos distribuidos en centros públicos, privados y privados concertados, durante el año académico 2000-2001; del total se eligió una muestra representativa de 139 sujetos.

En este caso concreto, por economía de tiempo y ante la imposibilidad de acceder a todos los componentes de la investigación, se tomó aleatoriamente un grupo de profesores y se midió la característica que interesaba estudiar sólo en ese grupo. Por lo tanto, se trabajó a partir de una muestra.

El tipo de muestreo empleado fue el probabilístico aleatorio (García, Pérez y Del Río, 1992), método que tiene como rasgo distintivo la extracción de los elementos de la población realizada al azar, de acuerdo con el procedimiento que se considere oportuno. En esta ocasión, se utilizó la generación de números aleatorios por ordenador a través del programa del SPSS. Las cifras extraídas de modo casual a partir de este método fueron 139 sujetos, distribuidos de la siguiente forma: 60 sujetos (43\%) procedentes de 11 centros públicos y un total de 79 sujetos $(57 \%)$ de 14 centros privados y concertados y 1 centro privado (Buendía y otros, 1999). Este número de docentes representaba el 60\% de la población de estudio; 
M. ${ }^{a}$ CARMEN PEREIRA DOMINGUEZ Y M. ${ }^{a}$ VICTORIA MARÍN VALLE

RESPUESTAS DOCENTES SOBRE EL CINE COMO PROPUESTA PEDAGÓGICA.

ANÁLISIS DE LA SITUACIÓN EN EDUCACIÓN SECUNDARIA

por consiguiente, creemos que los resultados obtenidos se consideran significativos de la realidad en la que se desarrolla la tarea docente del profesorado que trabaja en dicho nivel.

\section{VARIABLES ESTUDiadAS}

Para la realización de este estudio, se eligieron un total de 20 variables, agrupadas en tres grandes bloques:

1. Variables de identificación: recogen información de la muestra sobre los datos personales (género y edad), contextuales (tipo de centro) y docentes (dedicación).

2. Variables sobre los medios audiovisuales en general: recaban datos sobre el equipamiento y la utilización de los medios audiovisuales:

- Variable de recursos didácticos de que se dispone: retroproyector, proyector de diapositivas, televisión, vídeo, cañón de vídeo, DVD, ordenador, casete, audio CD, proyector de opacos, cámara de vídeo, proyector de películas, prensa, etc.

- Variable de utilización: frecuencia de uso y cantidad de los medios audiovisuales.

3. Variables sobre el uso de cine: consiguen información acerca de la utilización del cine; la actitud o criterio de los docentes sobre el tema a investigar; la utilización, el conocimiento y el juicio del profesorado con respecto a la estrategia grupal del cine forum; la actitud y la opinión de los profesores sobre la necesaria formación específica del tema a tratar y la aplicación y el conocimiento de determinadas películas.

\section{INSTRUMENTO DE MEDIDA}

La recogida de información sobre los medios audiovisuales en general y del cine en particular, se llevó a cabo a través de la aplicación de un cuestionario confeccionado para tal efecto.

Este instrumento se estructuró a modo de cuestionario combinando preguntas abiertas y cerradas. Su diseño pasó por distintas etapas con las consiguientes modificaciones, tanto en el formato externo como en la formulación de las preguntas, hasta obtener el modelo deseado (Visauta, 1989; Arnal y otros, 1992; Latorre y otros, 1996). Para ello, se tuvieron en cuenta los siguientes parámetros: que su extensión no sobrepasara los 25 ítems, que éstos no resultaran repetitivos, que siguieran un orden lógico y que las preguntas constituyeran baterías o bloques temáticos. 
M. ${ }^{a}$ CARMEN PEREIRA DOMÍNGUEZ Y M. ${ }^{a}$ VICTORIA MARÍN VALLE RESPUESTAS DOCENTES SOBRE EL CINE COMO PROPUESTA PEDAGÓGICA. ANÁLISIS DE LA SITUACIÓN EN EDUCACIÓN SECUNDARIA

Por tanto, el cuestionario elaborado consta de 20 preguntas organizadas según tres grandes bloques: el primero comprende los datos de identificación (items de 1 a 4), el segundo incluye los datos relativos al uso de los medios audiovisuales (items 5 y 6) y el tercero se atiene a los datos referidos al cine (items de 7 a 20).

Una vez delimitada la información que se pretendía recoger con la aplicación del cuestionario, el tipo de preguntas a realizar, la formulación de las mismas, el número y su ordenación, se procedió a una prueba piloto en la que participaron 20 personas, con el objeto de comprobar el correcto funcionamiento del mismo como instrumento o técnica de recogida de información. Esta prueba piloto permitió verificar si el enunciado de las preguntas era exacto y comprensible y si la extensión era la adecuada; además de constatar si se evidenciaban resistencias psicológicas o fatigas en las personas encuestadas. Una vez subsanados los errores que se pudieran detectar a través del estudio piloto, se dio paso a la confección definitiva del cuestionario.

Posteriormente, se redactó una carta de presentación, protocolaria, dirigida a los cargos directivos de cada uno de los centros seleccionados. Ésta reflejaba fundamentalmente el objetivo de la investigación y la imprescindible colaboración de los docentes de dichos centros, así como el agradecimiento por haber participado en el proyecto.

La vía seguida para la obtención de información se canalizó por medio de la entrevista personal. A pesar de que esta técnica de aplicación requería mayor tiempo y dedicación, debido a los continuos desplazamientos y reuniones mantenidas con los directores y docentes participantes, el esfuerzo quedó compensado al comprobar el alto grado de fiabilidad y respuestas conseguidas, sobre todo si se contrasta con otros sistemas vigentes como el cuestionario por correo, la encuesta telefónica y demás afines.

\section{ANÁlISIS DE LOS DATOS OBTENIDOS}

El examen de datos se llevó a cabo a través de técnicas que permitieron los análisis descriptivos y correlacionales. Para ello, se utilizó el paquete estadístico SPSS, versión 10.0 para Windows que facilitó la obtención de los siguientes resultados.

Las variables de identificación nos indican que predomina el género femenino, con 83 mujeres (59,7\%), frente al masculino, con 56 varones, lo que supone un $(40,3 \%)$ del total de la muestra.

Por lo que respecta a la edad, se observa en las respuestas del profesorado participante que la mayoría se encuentran entre los 31-50 años; siendo minoritaria la representación de profesores pertenecientes al último intervalo, es decir, de 61 años en adelante.

Sobre los datos correspondientes al contexto, de los 139 sujetos que componen dicha muestra, 60 (43\%) proceden de centros públicos y 79 (57\%), de centros 
privados concertados. De ello deducimos que esta distribución del profesorado es equitativa.

Otra de las preguntas formuladas en los datos de identificación es la concerniente a la dedicación docente. Se trata de indagar sobre la(s) materia(s) que impartían cada uno de los profesores implicados en este estudio.

De los resultados obtenidos, observamos que existe un elevado porcentaje de docentes que imparten clase en el área de lengua, mientras que el resto de los encuestados están vinculados a las áreas de matemáticas, tecnología, música, educación física y plástica.

Los datos presentados a continuación recogen información sobre las variables relacionadas con los medios audiovisuales. Éstas comprenden la dotación y la frecuencia.

Con respecto a la dotación (ítem 5), se analiza el equipamiento existente: retroproyector, proyector de diapositivas, televisión, vídeo, películas cinematográficas, ordenador, CD audio, cámara de vídeo..., dejando abierta la posibilidad de adjuntar medios como el DVD, el cañón de vídeo y otros.

La lectura de los datos nos manifiesta que existe una mayor presencia de la televisión $(93,5 \%)$, seguida del vídeo $(93,5 \%)$, del ordenador $(86,3 \%)$ y de la prensa $(82,7 \%)$. A continuación, se encuentran el proyector de diapositivas $(82,01 \%)$, el retroproyector $(76,9 \%)$, el casete $(75,5 \%)$ y las películas $(61,8 \%)$, encontrándose además varias unidades en los centros educativos.

Los demás medios audiovisuales tienen menor presencia, así como el número de unidades en los centros también resulta inferior.

Cabe señalar los datos respecto al desconocimiento que el profesorado posee acerca de los medios de que dispone en su centro al responder "no sé".

Con referencia a la variable frecuencia del uso de los medios (ítem 6), se puede afirmar que los profesores participantes en el estudio no suelen ser usuarios habituales de los medios audiovisuales, en general. Según los datos obtenidos se constata como, a excepción del retroproyector y la prensa, cuyos porcentajes más altos se localizan en la categoría "de vez en cuando", el resto de los medios obtienen un mayor índice en la categoría "nunca". 
M. - CARMEN PEREIRA DOMÍNGUEZ Y M. ․ VICTORIA MARÍN VALLE RESPUESTAS DOCENTES SOBRE EL CINE COMO PROPUESTA PEDAGÓGICA.

ANÁLISIS DE LA SITUACIÓN EN EDUCACIÓN SECUNDARIA

TABLA 1

DENSIDAD DE MEDIOS AUDIOVISUALES

\begin{tabular}{|c|c|c|c|c|c|c|}
\hline & \multicolumn{2}{|c|}{ RETROPROYECTOR } & \multicolumn{2}{|c|}{ PROYECTOR DIAPOSITIVAS } & \multicolumn{2}{|c|}{ TELEVISIÓN } \\
\hline & RECUENTO & $\%$ & RECUENTO & $\%$ & RECUENTO & $\%$ \\
\hline No sé & 28 & 20,1 & 22 & 15,8 & 9 & 6,5 \\
\hline & 4 & 2,9 & 3 & 2,2 & & \\
\hline $1-2$ & 83 & 59,7 & 73 & 52,5 & 71 & 51,1 \\
\hline $3-4$ & 20 & 14,4 & 29 & 20,9 & 50 & 36,0 \\
\hline 5 o más & 4 & 2,9 & 12 & 8,6 & 9 & 6,5 \\
\hline \multirow[t]{3}{*}{ TOTAL } & 139 & 100,0 & 139 & 100,0 & 139 & 100,0 \\
\hline & \multicolumn{2}{|c|}{ VÍDEO } & \multicolumn{2}{|c|}{ ORDENADOR } & \multicolumn{2}{|c|}{ CASETE } \\
\hline & RECUENTO & $\%$ & RECUENTO & $\%$ & RECUENTO & $\%$ \\
\hline No sé & 9 & 6,5 & 18 & 12,9 & 26 & 18,7 \\
\hline & & & 1 & 0,7 & 8 & 5,8 \\
\hline $1-2$ & 81 & 58,3 & 36 & 25,9 & 28 & 20,1 \\
\hline $3-4$ & 44 & 31,7 & 23 & 16,5 & 26 & 18,7 \\
\hline 50 más & 5 & 3,6 & 61 & 43,9 & 51 & 36,7 \\
\hline \multirow[t]{3}{*}{ TOTAL } & 139 & 100,0 & 139 & 100,0 & 139 & 100,0 \\
\hline & \multicolumn{2}{|c|}{ PROYECTOR OPACOS } & \multicolumn{2}{|c|}{ CÁMARA VÍDEO } & \multicolumn{2}{|c|}{ CINE (PELÍCULAS) } \\
\hline & RECUENTO & $\%$ & RECUENTO & $\%$ & RECUENTO & $\%$ \\
\hline No sé & 88 & 63,3 & 66 & 47,5 & 45 & 32,4 \\
\hline 0 & 19 & 13,7 & 25 & 18,0 & 8 & 5,8 \\
\hline $1-2$ & 27 & 19,5 & 45 & 32,4 & 21 & 15,1 \\
\hline $3-4$ & 2 & 1,4 & 1 & 0,7 & 12 & 8,6 \\
\hline 5 o más & 3 & 2,2 & 2 & 1,4 & 53 & 38,1 \\
\hline \multirow[t]{3}{*}{ TOTAL } & 139 & 100,0 & 139 & 100,0 & 139 & 100,0 \\
\hline & \multicolumn{2}{|c|}{ PRENSA } & \multicolumn{2}{|c|}{ DVD } & \multicolumn{2}{|c|}{ CAÑON } \\
\hline & RECUENTO & $\%$ & RECUENTO & $\%$ & RECUENTO & $\%$ \\
\hline No sé & 21 & 15,1 & 9 & 7 & 10 & 7,1 \\
\hline 0 & 3 & 2,2 & 121 & 87 & 121 & 87,0 \\
\hline $1-2$ & 31 & 22,3 & 7 & 5 & 6 & 4,9 \\
\hline $3-4$ & 51 & 36,7 & 2 & 1 & 2 & 1,0 \\
\hline 5 o más & 33 & 23,7 & & & & \\
\hline TOTAL & 139 & 100,0 & 139 & 100 & 139 & 100,0 \\
\hline
\end{tabular}


M. ${ }^{a}$ CARMEN PEREIRA DOMÍNGUEZ Y M. ${ }^{a}$ VICTORIA MARÍN VALLE RESPUESTAS DOCENTES SOBRE EL CINE COMO PROPUESTA PEDAGÓGICA ANÁLISIS DE LA SITUACIÓN EN EDUCACIÓN SECUNDARIA

TABLA 2

FRECUENCIA DE USO

\begin{tabular}{|c|c|c|c|c|c|c|}
\hline & \multicolumn{2}{|c|}{ RETROPROYECTOR } & \multicolumn{2}{|c|}{$\begin{array}{l}\text { PROYECTOR } \\
\text { DIAPOSITIVAS }\end{array}$} & \multicolumn{2}{|c|}{ VÍDEO } \\
\hline & RECUENTO & $\%$ & RECUENTO & $\%$ & RECUENTO & $\%$ \\
\hline Todos los clías & & & 1 & 0,7 & & \\
\hline 1-2 veces por semana & 4 & 2,9 & 6 & 4,3 & & \\
\hline De vez en cuando & 94 & 67,6 & 51 & 36,7 & 48 & 34,5 \\
\hline Nunca & 41 & 29,5 & 81 & 58,3 & 91 & 65,5 \\
\hline TOTAL & 139 & 100,0 & 139 & 100,0 & 139 & 100,0 \\
\hline & \multicolumn{2}{|c|}{ TELEVISIÓN } & \multicolumn{2}{|c|}{ ORDENADOR } & \multicolumn{2}{|c|}{ CASETE } \\
\hline & RECUENTO & $\%$ & RECUENTO & $\%$ & RECUENTO & $\%$ \\
\hline Todos los días & & & 5 & $\overline{3,6}$ & 16 & 11,5 \\
\hline $1-2$ veces por semana & & & 16 & 11,5 & 18 & 12,9 \\
\hline De vez en cuando & 64 & 46,0 & 35 & 25,2 & 30 & 21,6 \\
\hline Nunca & 75 & 54,0 & 83 & 59,7 & 75 & 54,0 \\
\hline \multirow[t]{3}{*}{ TOTAL } & 139 & 100,0 & 139 & 100,0 & 139 & 100,0 \\
\hline & \multicolumn{2}{|c|}{$\begin{array}{c}\text { PROYECTOR } \\
\text { OPACOS }\end{array}$} & \multicolumn{2}{|c|}{$\begin{array}{c}\text { CÁMARA } \\
\text { FOTOGRÁFICA }\end{array}$} & \multicolumn{2}{|c|}{$\begin{array}{l}\text { CÁMARA } \\
\text { VÍDEO }\end{array}$} \\
\hline & RECUENTO & $\%$ & RECUENTO & $\%$ & RECUENTO & $\%$ \\
\hline Todos los días & & & & & & \\
\hline $1-2$ veces por semana & 2 & 1,4 & 1 & 0,7 & 1 & 0,7 \\
\hline De vez en cuando & 7 & 5,0 & 19 & 13,7 & 11 & 7,9 \\
\hline Nunca & 130 & 93,5 & 119 & 85,6 & 127 & 91,4 \\
\hline \multirow[t]{3}{*}{ TOTAL } & 139 & 100,0 & 139 & 100,0 & 139 & 100,0 \\
\hline & \multicolumn{2}{|c|}{ CINE (PELÍCULAS) } & \multicolumn{2}{|c|}{ PRENSA } & & \\
\hline & RECUENTO & $\%$ & RECUENTO & $\%$ & & \\
\hline Todos los días & & & 8 & 5,8 & & \\
\hline $1-2$ veces por semana & & & 17 & 12,2 & & \\
\hline De vez en cuando & 63 & 45,3 & 73 & 52,5 & & \\
\hline Nunca & 76 & 54,7 & 41 & 29,5 & & \\
\hline TOTAL & 139 & 100,0 & 139 & 100,0 & & \\
\hline
\end{tabular}


M." CARMEN PEREIRA DOMÍNGUEZ Y M." VICTORIA MARÍN VALLE RESPLESTAS DOCENTES SOBRE EL CINE COMO PROPUESTA PEDAGÓGICA. ANÁLISIS DE LA SITUACIÓN EN EDUCACIÓN SECUNDARIA

Del estudio de las tablas deducimos que el retroproyector y la prensa son los medios más comunes para el profesorado, con un porcentaje de utilización que ronda el $70 \%$, empleándolo en algún momento.

Los medios como la televisión, el casete, las películas de cine, el proyector de diapositivas y el ordenador tienen su rango de utilización en torno al 43\%. Un poco más atrás queda el uso del vídeo, en torno al 35\%, y totalmente descolgados el retroproyector de opacos, la cámara de vídeo y la cámara fotográfica, con porcentajes de utilización próximos al 10\%.

Podemos observar, a la vista de los resultados, que los medios menos manejados por los docentes son: el retroproyector de opacos que es desconocido para un $93,5 \%$, la cámara de vídeo para un 91,4\% y la cámara fotográfica para un $85,6 \%$.

Los datos expuestos a continuación plasman la información sobre las variables referentes a la utilización del cine entre los docentes de diferente género y edad que imparten docencia en centros de distinto carácter, sobre las peliculas, sobre la técnica grupal del cine forum y sobre las diversas vias de formación del profesorado.

Con respecto a las variables de utilización del cine damos respuesta a las cuestiones asociadas al uso o no uso de este medio por parte del profesorado, el por qué de su utilización, o no utilización, y las creencias relativas a tal uso (items 7, 8, 9 y 10).

La respuesta correspondiente a la primera cuestión, se comprueba en los siguientes porcentajes: 47 sujetos $(33,8 \%)$ dicen emplear el cine en su tarea docente y 92 sujetos $(66,2 \%)$, manifiestan el desuso del cine en sus actividades escolares.

En esta pregunta, destaca un considerable porcentaje de respuestas que nos permite verificar dos de las hipótesis iniciales de la investigación, donde se confirma que la utilización del cine es escasa dentro de la práctica docente y la inexistencia de diferencias significativas en cuanto a su uso, según las tipologías de centros, el género y la edad de los docentes participantes en la muestra.

De las razones que inclinan a los docentes a servirse o no del cine, se destacan las siguientes: ayuda a clarificar conceptos $(61,7 \%)$; facilita el incremento de información sobre un tema determinado $(61,7 \%)$; produce cambios de actitudes $(61,7 \%)$ y favorece el desarrollo de habilidades sociales $(59,6 \%)$.

En lo concerniente a la pregunta abierta sobre las razones del uso del cine incluida en este ítem, fue contestada por dos sujetos de la muestra, los cuales anotaron como una posible justificación el hecho de que el cine formativo puede llegar a sensibilizar al alumnado de cara a un tema de enseñanza.

En relación a la información obtenida acerca de los motivos principales por los que el profesorado no emplea habitualmente el cine dentro de su práctica docente, comprobamos que un elevado porcentaje de docentes resalta las siguientes puntualizaciones: no se usa ante la inexistencia de un espacio ni tiempo adecuado para trabajar con él en el aula $(69,6 \%)$, existe falta de motivación (63\%), se precisa una formación especializada del profesorado $(59,8 \%)$ y se evidencia escasez de tiempo e inflexibilidad en los horarios escolares (63\%).

A la pregunta abierta referida a los motivos por los que el profesorado no frecuenta el cine respondieron siete sujetos, de los cuales dos de ellos, afirman que 
M. ${ }^{a}$ CARMEN PEREIRA DOMINGUEZ Y M. ${ }^{a}$ VICTORIA MARÍN VALIE

RESPUESTAS DOCENTES SOBRE EL CINE COMO PROPUESTA PEDAGÓGICA.

ANÁLISIS DE LA SITUACIÓN EN EDUCACIÓN SECUNDARIA

otra razón añadida es el hecho de impartir clases a varios grupos del mismo nivel. En cuanto al resto de las respuestas, reflejan el escaso material educativo existente para trabajar con este medio.

Con referencia a los datos sobre las creencias asociadas en torno al cine, las respuestas que presentan un mayor índice de porcentaje fueron las siguientes: el cine ayuda a desarrollar habilidades sociales $(77,7 \%)$ y posibilita la adquisición de conocimientos $(76,3 \%)$.

Asimismo, el profesorado afirma que si se hace hincapié para obtener la máxima rentabilidad educativa de este medio, es necesario que el alumnado posea ciertos conocimientos básicos cinematográficos $(75,5 \%)$.

Según la información conseguida de esta muestra, apreciamos cómo la mayoría de los docentes tiene una visión positiva sobre el cine. A pesar de no ser un recurso familiar de modo habitual en su práctica educativa, se constata que los índices de mayor porcentaje se localizan en los intervalos de "muy de acuerdo" y "de acuerdo".

Una vez realizada la revisión de datos sobre la utilización del cine, exponemos a continuación la variable referida a la filmografía. Para ello, tratamos de recabar información sobre el empleo de películas en el área donde los docentes tienen docencia.

Tras una búsqueda y selección minuciosa, se confeccionó un listado de películas que consideramos de interés para la intervención pedagógica (Kobal, 1990; Equipo Reseña, 1987-2000; Touriñán, 1997; Raposo, 2002), resultando las siguientes ${ }^{3}$ :

Rain man (Barry Levinson, 1988), Lloviendo piedras (Ken Loach, 1993), El club de los poetas muertos (Peter Weir, 1989), Patch Adams (Tom Shadyac, 1997), Philadelphia (Johnattan Demme, 1993), La verdad sobre perros y gatos (Michael Lehmamm, 1996), La misión (Roland Joffé, 1986), El cartero y Pablo Neruda (Michael Radford, 1994), El caso Winslow (David Mamet, 1999), Manolito gafotas (Miguel Albadalejo, 2000), La vida es bella (Roberto Benigni, 1997), Cielo de octubre (Joe Johnston, 1999), Ben Hur (William Wyler, 1959), Jesús de Nazaret (Franco Zefirelli, 1978), Shine, el resplandor de un genio (Scott Hicks, 1995), El principe de Egipto (Brenda Chapman, Steve Hickner, Simon Wells, 1998), El hombre sin rostro (Mel Gibson, 1993), Semillas de rencor (John Singleton, 1995) y El jorobado de Nôtre-Dame (Peter Medak, 1996).

3. Conviene señalar que algunas de estas películas son conocidas y trabajadas por el profesorado implicado en este estudio, y además, colaboran en diversos programas como: "Cine e Saúde", promocionado desde las Concejalías de Sanidad del Concello de Ourense y subvencionado por el Plan de Prevención de Drogas de la Xunta de Galicia (Ameijeiras, Villar, Pereira y Sueiro, 2000, 593-607; Pereira y Rodríguez, 2000; Villar y Pereira, 2000; Ameijeiras, Pereira y Villar, 2001, 104-105); "Cine y Educación en Valores", diseñado por el Centro de Documentación y Pedagogía de la Asociación de Prensa Juvenil, promovido por la Fundación de Ayuda a la Drogadicción (coordinado por Escámez); "Cinensino" subvencionado por la Consellería de Sanidade de la Xunta de Galicia (coordinado por Peña) y el "Proyecto Cine y Transversales" iniciativa de la Revista Padres y Maestros. Además, insertamos en la bibliografía algunas páginas web de interés sobre filmografía. 
M.- CARMEN PEREIRA DOMÍNGUEZ Y M. - VICTORIA MARÍN VALLE RESPUESTAS DOCENTES SOBRE EL CINE COMO PROPUESTA PEDAGÓGICA. ANÁLISIS DE LA SITUACIÓN EN EDUCACIÓN SECUNDARIA

TABLA 3

UTILIZACIÓN DE PELÍCULAS FORMATIVAS

\begin{tabular}{|c|c|c|c|c|c|c|}
\hline \multirow{2}{*}{ USO } & \multicolumn{2}{|c|}{ RAIMAN } & \multicolumn{2}{|c|}{ LLOVIENDO PIEDRAS } & \multicolumn{2}{|c|}{ EL CLUB DE LOS POETAS } \\
\hline & RECUENTO & $\%$ & RECUENTO & $\%$ & RECUENTO & $\%$ \\
\hline NO SÉ & 62 & 44,6 & 90 & 64,7 & 36 & 25,9 \\
\hline SÍ & 42 & 30,2 & 22 & 15,8 & 77 & 55,4 \\
\hline $\mathrm{NO}$ & 35 & 25,2 & 27 & 19,4 & 26 & 18,7 \\
\hline TOTAL & 139 & 100,0 & 139 & 100,0 & 139 & 100,0 \\
\hline \multirow{3}{*}{ USO } & \multirow{2}{*}{\multicolumn{2}{|c|}{$\begin{array}{l}\text { PATCH } \\
\text { ADAMS }\end{array}$}} & \multirow{2}{*}{\multicolumn{2}{|c|}{ PHILADELPHIA }} & \multirow{2}{*}{\multicolumn{2}{|c|}{$\begin{array}{l}\text { LA VERDAD SOBRE } \\
\text { PERRROS Y GATOS }\end{array}$}} \\
\hline & & & & & & \\
\hline & RECUENTO & $\%$ & RECUENTO & $\%$ & RECUENTO & $\%$ \\
\hline NO SÉ & 78 & 56,1 & 54 & 38,8 & 81 & 58,3 \\
\hline & 29 & 29,9 & 52 & 37,4 & 26 & 18,7 \\
\hline NO & 32 & 23,0 & 33 & 23,7 & 32 & 23,0 \\
\hline TOTAL & 139 & 100,0 & 139 & 100,0 & 139 & 100,0 \\
\hline \multirow{2}{*}{ USO } & \multicolumn{2}{|c|}{$\begin{array}{c}\text { LA } \\
\text { MISIÓN }\end{array}$} & \multicolumn{2}{|c|}{$\begin{array}{l}\text { EL CARTERO Y } \\
\text { PABLO NERUDA }\end{array}$} & \multicolumn{2}{|c|}{$\begin{array}{l}\text { EL CASO } \\
\text { WINSLOW }\end{array}$} \\
\hline & RECUENTO & $\%$ & RECUENTO & $\%$ & RECUENTO & $\%$ \\
\hline NO SÉ & 48 & 34,5 & 64 & 46,4 & 86 & 61,9 \\
\hline & 58 & 41,7 & 45 & 32,6 & 21 & 15,1 \\
\hline NO & 33 & 23,7 & 30 & 21,0 & 32 & 23,0 \\
\hline TOTAL & 139 & 100,0 & 139 & 100,0 & 139 & 100,0 \\
\hline \multirow{2}{*}{ USO } & \multicolumn{2}{|c|}{ MANOLITO GAFOTAS } & \multicolumn{2}{|c|}{ LA VIDA ES BELLA } & \multicolumn{2}{|c|}{ CIELO DE OCTUBRE } \\
\hline & RECUENTO & $\%$ & RECUENTO & $\%$ & RECUENTO & $\%$ \\
\hline NO SÉ & 46 & 33,1 & 31 & 22,5 & 80 & 58,0 \\
\hline & 56 & 40,3 & 81 & 58,7 & 23 & 16,7 \\
\hline NO & 37 & 26,6 & 27 & 18,8 & 36 & 25,4 \\
\hline TOTAL & 139 & 100,0 & 139 & 100,0 & 139 & 100,0 \\
\hline \multirow{2}{*}{ USO } & \multicolumn{2}{|c|}{ BEN HUR } & \multicolumn{2}{|c|}{ JESÚS DE NAZARET } & \multicolumn{2}{|c|}{ SHINE } \\
\hline & RECUENTO & $\%$ & RECUENTO & $\%$ & RECUENTO & $\%$ \\
\hline NO SÉ & 54 & 39,1 & 60 & 43,2 & 79 & 56,8 \\
\hline & 49 & 35,5 & 41 & 29,5 & 27 & 19,4 \\
\hline NO & 36 & 25,4 & 38 & 27,3 & 33 & 23,7 \\
\hline TOTAL & 139 & 100,0 & 139 & 100,0 & 139 & 100,0 \\
\hline \multirow[t]{2}{*}{ USO } & \multicolumn{2}{|c|}{$\begin{array}{l}\text { EL PRÍNCIPE } \\
\text { DE EGIPTO }\end{array}$} & \multicolumn{2}{|c|}{$\begin{array}{l}\text { EL HOMBRE } \\
\text { SIN ROSTRO }\end{array}$} & \multicolumn{2}{|c|}{$\begin{array}{l}\text { SEMILLAS DE } \\
\text { RENCOR }\end{array}$} \\
\hline & RECUENTO & $\%$ & RECUENTO & $\%$ & RECUENTO & $\%$ \\
\hline NOSÉ & 56 & 40,9 & 70 & 50,4 & 86 & 61,9 \\
\hline & 47 & 33,6 & 35 & 25,2 & 23 & 16,5 \\
\hline NO & 36 & 25,5 & 34 & 24,5 & 30 & 21,6 \\
\hline TOTAL & 139 & 100,0 & 139 & 100,0 & 139 & 100,0 \\
\hline ICO & $\begin{array}{l}\text { EL J } \\
\text { DE N }\end{array}$ & $\mathrm{ADO}$ & & & & \\
\hline & RECUENTO & $\%$ & & & & \\
\hline NO SÉ & 55 & 39,6 & & & & \\
\hline SI & 49 & 35,3 & & & & \\
\hline NO & 35 & 25,2 & & & & \\
\hline TOTAL & 139 & 100,0 & & & & \\
\hline
\end{tabular}


RESPUESTAS DOCENTES SOBRE EL CINE COMO PROPUESTA PEDAGÓGICA.

ANÁLISIS DE LA SITUACIÓN EN EDUCACIÓN SECUNDARIA

Efectuado el repaso general de los resultados, podemos afirmar que la mayoría de las películas eran desconocidas para el profesorado, haciendo hincapié que el porcentaje más alto de respuesta se sitúa en la opción "no sé", excepto en las películas como, La vida es bella (Roberto Benigni, 1997), El club de los poetas muertos (Peter Weir, 1989), La misión (Roland Joffé, 1986) y Manolito gafotas (Miguel Albadalejo, 2000) que fueron seleccionadas por un $58,7 \% ; 55,4 \% ; 41,7 \%$ y $40,3 \%$ respectivamente.

Referente a la pregunta abierta sobre con qué otras películas trabajarían, cabe subrayar que los docentes se decantaron por títulos como:

El nombre de la rosa (J. J. Annaud, 1986), Ulises (Mario Camerini, 1954), Alejandro Magno, (Robert Rosen, 1956), Los visitantes (J. M. Poiré, 1992), Espartaco (S. Kubrick, 1960), Pena de muerte (Tim Robins, 1995), American bistory (Tony Kaye, 1999), El abuelo (José L. Garci, 1998), Los últimos dias del Edén (John Mc. Tiernan, 1992), Amistad (S. Spielberg, 1997), Amadeus (Milos Forman, 1984), West side story (Jerome Robins y Robert Wise, 1961), El piano (Jane Champion, 1993), Farinelly (Gérard Corbian, 1994), Fantasía (Walt Disney, 1940), Excalibur (J. Boorman, 1981), Carros de fuego (H. Hudson, 1980), Ghandi (R. Attenborough, 1983), Hermano sol, bermana luna (Franco Zefirelli, 1972), Los miserables (Josce Dayan, 2000), La bella y la bestia (Linda Woolverton, 1991), El rey león (Roger Allen y Rob Minkoff, 1994), Barrio (Fernando León, 1998), El inolvidable Simón Birch (M. Steven Jonson, 1998), Mejor imposible (J. L. Brooks, 1997), Tierras de penumbra (R. Attenborough, 1994), Silkwood (Mike Nichols, 1983), Amistades peligrosas (Stephen Frears, 1988) y La princesa prometida (Bob Reiner, 1987).

Ante lo expuesto, se evidencia la calidad educativa de estas sugerencias cinematográficas recomendadas por los docentes de la muestra.

Si se analizan y correlacionan las variables del binomio películas-áreas, apreciamos que su utilización es más frecuente en el profesorado que imparte materias vinculadas al área de lengua, incluyendo literatura, lengua gallega e idiomas extranjeros, que en quienes lo hacen en las materias de matemáticas, música y educación física.

Por lo que respecta a las variables correspondientes a la técnica del cine forum, tratan de recabar información sobre cuestiones determinadas como la participación en la misma y las razones para su aplicación (ítems 12, 13, 14 y 15).

En cuanto a la participación de estos sujetos en alguna sesión de cine forum, los resultados plasman que: 83 sujetos $(59,7 \%)$ afirman haber colaborado en alguna sesión, frente a 56 sujetos $(40,3 \%)$ que no han asistido nunca a dicha actividad, ni de manera formal, ni informal. Sin embargo, como podemos comprobar, existe un alto porcentaje de docentes que valoran el cine como una estrategia de intervención pedagógica.

Otra de las cuestiones trata cómo trabajar o no con dicha técnica en el aula. La respuesta arrojó los siguientes datos: 99 sujetos consideran el cine forum como 
una técnica óptima para trabajar con ella en el aula $(71,2 \%)$, frente a 17 sujetos que manifiestan que nunca la utilizarían en el aula (12,2\%).

Los motivos por los cuales podrían emplear o no esta técnica dentro de su práctica docente fueron: que induce a cambios de actitudes $(70,3 \%)$, que favorece la participación activa del alumnado $(63,4 \%)$, que clarifica contenidos $(67 \%)$ y que responde a sus intereses (65\%).

A la pregunta abierta sobre la utilización de la estrategia del cine forum, contestaron cinco docentes, de los cuales cuatro afirman, de modo particular, que otra razón añadida es la motivación de los alumnos para realizar algo distinto y el quinto porque les estimula la memoria visual.

Entre las justificaciones para no trabajar el cine, destacan preferentemente las siguientes: se trata de una actividad compleja $(70,6 \%)$, existe falta de información para desarrollar esta técnica $(70,6 \%)$ y resulta una actividad de larga duración $(70,6 \%)$.

Las variables que analizan el uso del cine están ligadas a la formación de los docentes. Desde este prisma, se tocan aspectos como la preparación que debe tener el profesorado para el uso y conocimiento del medio (ítems 18 y 19); así como las personas responsables de impartir esta formación básica (ítem 17) y la necesidad de formarse en el caso de que este colectivo lo considere preciso (item 16).

En cuanto a si se estima conveniente o no la formación específica de los docentes para familiarizarse con el cine, la respuesta observada fue la siguiente: un $77 \%$ demandan la necesidad de una preparación específica del profesorado en el uso y conocimiento de este medio, frente a un 5\% que la consideran innecesaria.

También se indaga sobre qué organismo(s) asumiría(n) esta responsabilidad formativa: un $50,5 \%$ se inclinan por los centros de formación de profesores y recursos: un $23,4 \%$ señalan el centro educativo donde trabajan; un $15,8 \%$ citan a la universidad, y finalmente, un 10,3\% apuntan que es una función independiente y particular de cada docente.

A la pregunta abierta referente al tipo de instituciones encargadas de la formación, contestaron cuatro profesores, quienes coinciden en contemplar, como opción formativa, la oferta procedente de diversos cine-clubes o instituciones similares existentes en el entorno.

La última cuestión se corresponde con el interés de los docentes para formarse en el conocimiento y dominio de este medio (ítems 18 y 19). Las respuestas obtenidas fueron las siguientes: 102 profesores $(73,4 \%)$ manifestaron su curiosidad por formarse en el tema; y en cuanto al momento más adecuado para llevar a cabo esta formación, bien a través de cursos o jornadas, 78 docentes $(76,5 \%)$ se decantaron por el inicio del curso escolar.

El cuestionario concluye con una pregunta abierta, centrada en la reflexión personal sobre el cine. A través de ella constatamos algunos de los pensamientos emitidos por el grupo de docentes componentes de la muestra de estudio, que insertamos a continuación. Dichos pensamientos van desde la insistencia de considerar el cine como una asignatura pendiente, tanto para el profesorado como 
para el alumnado, argumentando que «Debería figurar como una materia formativa dentro de las ciencias de la comunicación", o que "Algunas jornadas o cursillos sobre estos temas animarían al profesorado a intentarlo, suelen ser los motores para cambiar de rutina”.

Además, algunos califican el cine de primordial para la formación permanente de la persona, es decir: “Los medios audiovisuales son unos instrumentos imprescindibles hoy para la formación del ser humano, en cualquiera de sus niveles de aprendizaje" o, también, "Lo utilizo como tutor en la tutoría para temas transversales, sirve de conexión con el tema a tratar", hasta quien afirma que el cine es innecesario, "Lo considero un recurso poco óptimo para el ámbito escolar".

Igualmente, hay quienes se acogen a programas de intervención pedagógica vigentes, a pesar de las múltiples dificultades de horarios y falta de espacios, de adaptación a las áreas curriculares determinadas, de motivación en el grupo-clase y comentan, "Llevamos varios años participando en el ciclo - Cine y Valores-, y nos parece muy positivo, pero podemos hacerlo y trabajarlo en el aula porque tenemos una asignatura específica para ello. De no ser así, entiendo que no pueda ser un medio muy habitual por falta de tiempo, y además, los alumnos no siempre están dispuestos a reflexionar y participar con seriedad y una mínima profundidad como sería deseable". Y siguen añadiendo: "No utilizo habitualmente el cine formativo por dificultades en la adaptación de horarios, espacios, repetición de cursos, etc., pero sí lo considero interesante. Lo utilizo ocasionalmente y a veces indirectamente — películas visionadas por el alumnado fuera del aula—.

Por último, mencionamos a aquellos docentes que ni se plantean la integración del cine en el currículo por juzgarlo de escaso interés e innecesario, con expresiones como: «El horario escolar no permite introducir fácilmente el cine en las aulas. Gran parte del profesorado y del alumnado considera que hacer esta actividad es perder clases" o, esta otra opinión: "Me parece interesante, no obstante, considero que ese tiempo se le podría dedicar a la lectura comprensiva. ¡Estaremos de acuerdo que cada vez se lee menos y, por el contrario, estamos mucho tiempo delante de - pantallas_ como la televisión, ordenador...!”.

\section{CONCLUSIÓN}

Plasmamos a continuación las consideraciones más relevantes de la investigación desarrollada:

- Los profesores pertenecientes a la muestra de estudio no son usuarios habituales de los medios audiovisuales.

- La información recabada sobre el uso del cine aporta resultados que muestran cómo los docentes aplican escasamente este recurso en los procesos de intervención pedagógica, con independencia de su sexo, edad o tipo de centro. 
M. ${ }^{\text {a }}$ CARMEN PEREIRA DOMINGGUEZ Y M. ${ }^{\text {a }}$ VICTORIA MARÍN VALLE RESPUESTAS DOCENTES SOBRE EL CINE COMO PROPUESTA PEDAGÓGICA ANÁLISIS DE LA SITUACIÓN EN EDUCACIÓN SECUNDARIA

- Del uso de este medio, el profesorado señala entre los efectos positivos que para su tarea educativa se derivan los siguientes: que ayuda a clarificar contenidos, que incrementa la información relacionada con algún tema concreto, que facilita el desarrollo de habilidades sociales y que favorece la clarificación de valores y los cambios de actitud.

- Entre los motivos que justifican el escaso uso de este medio en el aula, los docentes coinciden en que carecen de espacios apropiados y del tiempo idóneo para trabajar el cine en el aula. Por tanto, ni se encuentran motivados para ello, ni tampoco la distribución de actividades en el horario escolar tiene, en muchos casos, la conveniente flexibilidad para realizar actividades de este tipo.

- El profesorado expresa que si el alumnado poseyera conocimientos de lenguaje cinematográfico, los procesos de aprendizaje desarrollados y acompañados de este medio audiovisual, obtendrían mejores resultados.

- También indican que las películas con cierta calidad educativa promueven la observación, el análisis, la reflexión y la mejora de variados aspectos relevantes para la formación de las personas, como el ciclo de la vida; los cambios personales, sociales e institucionales; los derechos humanos; la clarificación de valores culturales, humanos, técnico-científicos o artísticos; los procesos de aprendizaje y de intervención pedagógica, entre otros.

- Aunque la mayoría de las películas seleccionadas en el estudio eran desconocidas para los docentes, sin embargo, las más reconocidas por orden de prioridad resultaron: La vida es bella (Roberto Begnini, 1997), El club de los poetas muertos (Peter Weir, 1989), La misión (Roland Joffé, 1986) y Manolito gafotas (Miguel Albadalejo, 2000). En dicha selección destacaron como rasgos comunes, el éxito comercial de las mismas y las campañas publicitarias de las que fueron objeto.

- El cine es más utilizado por el profesorado que imparte clases en áreas como sociales, lengua y literatura, y formación religiosa y ética; y menos por el vinculado a las áreas de matemáticas, música y educación física.

- En cuanto a la técnica del cine forum, aunque un sector importante de la muestra manifestó haber participado en alguna sesión de cine forum, ya sea de manera formal o informal, consideran que es una estrategia de intervención pedagógica óptima para trabajar con ella en clase puesto que incrementa la participación activa, favorece la clarificación de valores y mejora los cambios de actitudes, facilita la resolución de conflictos y responde a los intereses particulares del alumnado.

- Algunos docentes demandan una preparación en actitudes adecuadas para aplicar el cine forum en el aula porque: se trata de una actividad compleja, de duración amplia, faltan materiales educativos de apoyo y se precisa información, comprensión y dominio, tanto por parte del profesorado como del alumnado. 
- Se constata la necesidad de sensibilizar y formar de modo permanente al profesorado ante este tipo de temas, reconsiderando y mejorando los inconvenientes por ellos expuestos.

El estudio llevado a cabo ha cumplido una primera fase que seguirá con otras que se desarrollarán y profundizarán. Por tanto, sin pretender concluir, exponemos además algunas alternativas educativas que posibiliten la intervención pedagógica con el cine:

- Consideramos necesario sensibilizar, motivar y responsabilizar al profesorado de la importancia de optimizar los procesos de aprendizaje desarrollados a través del cine. Aunque para ello, hace falta un cambio en las demandas de la sociedad, de las autoridades educativas, de los responsables de los centros, de la participación de las familias, de la dotación de materiales educativos de apoyo, de la utilización de espacios adecuados y de la flexibilización de horarios para incrementar el trabajo interdisciplinar, etc. Cubiertas estas demandas, posiblemente los docentes encuentren sentido a la innovación, a la acción-reflexión-acción, al trabajo en equipo, a la recreación constante de su tarea educativa, etc.

- Es conveniente no limitar el cine formativo a las películas bien valoradas por los expertos, a pesar del concepto señalado en párrafos anteriores. Quizá resulte aconsejable ver buen cine para crear afición y su valor en sí, pero también, ver cine normal que tiene éxito para ayudar a verlo de forma distinta, con una mirada más inteligente.

- También nos parece interesante insistir en la oportunidad que ofrecen los filmes para retomar las preguntas latentes en la vida del alumnado y ayudarles a distanciarse, enjuiciarlas, valorarlas, ver la posibilidad de plantear alternativas o respuestas diferentes. Frente a los amplios espacios de tiempo dedicados por los adolescentes ante el televisor que puede cobrar una importancia superior a la del centro educativo, el profesorado o los libros y actividades explícitamente didácticas.

- En resumen, se trata de establecer maneras de conocer y analizar las vías de conocimiento organizado que no sólo contemplen la reproducción del escenario cultural, sino también la reconstrucción reflexiva y crítica. 


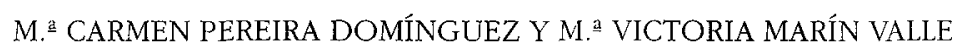
RESPUESTAS DOCENTES SOBRE EL CINE COMO PROPUESTA PEDAGÓGICA. ANÁLISIS DE LA SITUACIÓN EN EDUCACIÓN SECUNDARIA

\section{BiBliografía}

ALCOVER, N. y ÚRBEZ, L. (1976) Introducción a la lectura crítica del film. Barcelona, Don Bosco.

ALONSO, M. ${ }^{a}$ L. y PEREIRA, M. ${ }^{a}$ C. (2000) El cine como medio-recurso para la educación en valores. Un enfoque teórico y tecnológico, Pedagogía Social. Revista Interuniversitaria. Monográfico «Educación Social y Medios de Comunicación", 5, 2.época, 127-147.

AMEIJEIRAS, S.; VILLAR, P.; PEREIRA, M.ํㅡㄹ C. y SUEIRO, E. (2000) Cine y salud. Aproximación a un programa de acción socio-educativa y cultural en los ámbitos formal y no formal, en CARIDE, J. A. (coord.). Educación social y políticas culturales. Santiago de Compostela, Servicio de publicaciones de la Universidad de Santiago de Compostela.

AMEIJEIRAS, S.; PEREIRA, M. ${ }^{a}$ C. y VILLAR, P. (2001) Evaluación y análisis de una propuesta de intervención pedagógica en educación y valores. El Programa "Cine y Salud" del Ayuntamiento de Ourense, en NÚÑEZ CUBERO, L. y otros (eds.). Evaluación de politicas educativas. Huelva, Servicio de Publicaciones de la Universidad de Huelva.

ARNAL, J.; DEL RINCÓN, D. y LATORRE, A. (1992) Investigación educativa. Fundamentos y metodología. Barcelona, Labor.

BODGAN, R. y BIKLEN, S. (1982) Qualitative research for education. An introduction to theory and methods. Boston, Allyn and Bacon.

BUENDÍA, L.; GONZÁLEZ, D.; GUTIÉRREZ, J. y PELAGAGAR, M. (1999) Modelos de análisis de la investigación educativa. Sevilla, Alfar.

BUXARRAịs, M. ․ R.; MARTÍNEZ, M.; PUIG, J. M. ${ }^{a}$ y TRILLA, J. (1995) La educación moral en Primaria y Secundaria. Zaragoza, Edelvives.

CENTRO DE COMUNICACIÓN Y PEDAGOGÍA (1997-2002) Guías Didácticas Cinematográficas. Barcelona, Asociación Prensa Juvenil.

COHEN, L. y MANIÓN, L. (1990) Métodos de investigación educativa. Madrid, La Muralla, 73.

COLL, M.; SELVA, M. y SOLÁ, A. (1995) Monográfico. Cien años de cine, Revista Cuadernos de Pedagogía, 242, 8-54.

COPPEN, H. (1982) Utilización didáctica de los medios audiovisuales. Madrid, Anaya.

DIOS, M. (2001) Cine para convivir. Santiago de Compostela, Toxo Soutos, 33-41.

ELLIOT, J. (1990) La investigación-acción en educación. Madrid, Morata, 23.

EQUIPO RESEÑA (1987-2000) Cine para leer. Bilbao, Mensajero.

ESCÁMEZ, J. (coord.) (1996) Cine y valores. Madrid, Fundación de Ayuda a la Drogadicción.

ESCAMEZ, J. y ORTEGA, P. (1989) La enseñanza de actitudes y valores. Valencia, Nau Llibres.

ESPELT, R. (2001) Jonás cumplió los 25. La educación formal en el cine de ficción 19752000. Barcelona, Alertes, 24.

FERNÁNDEZ SEBASTIÁN, J. (1989) Cine e bistoria en el aula. Madrid, Akal.

GARCÍA, J. L.; PÉREZ, R. y DEL RÍO, D. (1992) Problemas y diseños de investigación resueltos. Madrid, Dykinson.

GONZÁLEZ MARTEL, J. (1996) El cine en el universo de la ética. El cine-forum. Madrid, Anaya.

GUBERN, R. (1995) Historia del cine. Barcelona, Lumen.

JEANNE, R. y FORD, C. (1988) Historia ilustrada del cine. Madrid, Alianza, 11-24.

KOBAL, J. (1990) Las 100 mejores peliculas. Madrid, Alianza.

LAMET, P. M. (coord.) (1991) Amigos del cine. Zaragoza, Edelvives. 
M. ${ }^{\underline{a}}$ CARMEN PEREIRA DOMÍNGUEZ Y M. ${ }^{a}$ VICTORIA MARÍN VALLE

RESPUESTAS DOCENTES SOBRE EL CINE COMO PROPUESTA PEDAGÓGICA.

ANÁLISIS DE LA SITUACIÓN EN EDUCACIÓN SECUNDARIA

LATORRE, A. y otros (1996) Bases metodológicas de la investigación educativa. Barcelona, GR 92.

LOSCERTALES, F. y NÚÑEZ, T. (2001) Violencia en las aulas. Barcelona, Octaedro, 7-12.

LUMET, S. (1999) Asi se hacen las peliculas. Barcelona, Rialp.

MAKING OF (2001) Cuadernos de Cine y Educación, 3.

MARÍN, M. ${ }^{a}$ V. y PEREIRA, M. ${ }^{a}$ C. (2000) Cine y Transversales "La vida es bella", Revista Padres y Maestros, 256, 113-116.

ORTEGA, P.; GIL, R. y MÍNGUEZ, R. (1997) Valores y educación. Barcelona, Ariel.

PEÑA, I. (dir.) (1999-2002) Programa de prevención de drogodependencias. O cinema no ensino. Bilbao, Irudi Biziak-Santiago de Compostela, Xunta de Galicia.

PEREIRA, M. ․ C. y RODRÍGUEZ, R. M. (2000) Cine e Saide. Chovendo Pedras. Ourense, Imprenta San Martín. Santiago de Compostela, Xunta de Galicia.

PLATAS, A. M.. (coord.) (1994) Literatura, cine, sociedad. A Coruña, Tambre, 7-9.

RAPOSO, M. (2002) Novas tecnoloxías aplicadas á educación: aspectos técnicos e didácticos. Vigo, Servicio de Publicacións da Universidade de Vigo, 125-148.

RODRÍGUEZ NEIRA, T. (1999) La cultura contra la escuela. Barcelona, Ariel, 78-79.

TORRE, S. DE LA (1996) Cine formativo. Barcelona, Octaedro, 9-12.

TOURIÑÁN, J. M. (1997) La racionalidad de la intervención pedagógica: explicación y comprensión, Revista de Educación, 314, 157-186.

URPÍ, C. (2000) La virtualidad educativa del cine. A partir de la teoría filmica de Jean Mitry (1904-1988). Pamplona, Eunsa, 258.

VV.AA. (1997) La tolerancia en el cine. París, Unesco.

- (1997-2002) Páginas centrales "Cine y Transversales", Revista Padres y Maestros.

VÁZQUEZ FREIRE, M. (1999) Cine e dereitos humanos, Revista Galega de Educación. Monográfico "Educación e Dereitos Humanos", 33, 56-67.

VÁZQUEZ GÓMEZ, G. (1994) Dimensión teórico-práctica de la educación, en CASTILLEJO, J.; VÁZQUEZ, G.; COLOM, A. J. y SARRAMONA, J. Teoría de la educación. Madrid, Taurus, $45-55$.

VILLAR, P. y PEREIRA, M. ${ }^{a}$ C. (2000) Cine e Saúde. O Caso Winslow. Ourense, Imprenta San Martín. Santiago de Compostela, Xunta de Galicia.

YING, A. (1987) Case study research. Desing and methods. Beverly Hills, Sage publications, 13.

VISAUTA, B. (1989) Técnicas de investigación social. Tomo I: Recogida de datos. Barcelona, Promociones y Publicaciones Universitarias, S.A. 